\title{
Appreciating diversity of goals in computational neuroscience
}

\author{
Konrad Paul Kording ${ }^{1}$, Gunnar Blohm ${ }^{2}$, Paul Schrater ${ }^{3}$, Kendrick Kay ${ }^{4}$ \\ ${ }^{1}$ Departments of Bioengineering and Neuroscience, University of Pennsylvania, Philadelphia, PA, USA \\ ${ }^{2}$ Centre for Neuroscience Studies, Queen's University, Kingston, ON, Canada \\ ${ }^{3}$ Departments of Psychology and Computer Science, University of Minnesota, Minneapolis, MN, USA \\ ${ }^{4}$ Center for Magnetic Resonance Research (CMRR), Department of Radiology, University of Minnesota, Minneapolis, MN, USA
}




\begin{abstract}
Outsiders often perceive computational neuroscience as a coherent field that strives towards progressively better models of the brain. However, informal interactions with modelers often reveal wildly divergent goals. Here we explicitly address the diversity of goals that motivate and ultimately influence modeling efforts. We argue that a wide range of goals can be meaningfully taken to be of highest importance. A simple informal survey conducted on the Internet confirmed the diversity of goals in the community. We propose that many disagreements in evaluating the merit of computational research stem from differences in goals and not from the mechanics of constructing, describing, and validating models. We suggest that authors should state explicitly their goals when proposing models so that others can judge the quality of the research with respect to its goals.
\end{abstract}




\section{Main text}

\section{Diversity of modeling goals}

Models are essential for progress in neuroscience and exist in a variety of forms and flavors. Models can summarize existing data. They can help forge links between brain and behavior. They can guide and inspire experiments and clinical treatments. They can help uncover hidden assumptions and help assess the suitability of hypothesized relationships. They can provide mathematical insights and can lead to systems that solve real-world problems. Accordingly, there is a large community of neuroscientists who construct and use models.

As computational neuroscientists, we became interested in the goals of modeling when we noticed stark differences across models in different papers and fields of neuroscience. For example, when Kendrick studies nonlinearities in the human brain, he cares most about macroscopic measurements and model interpretability. When Gunnar writes a paper about linear-systems explanations of eye movements, he cares most about behavior, mathematical simplicity, and the real-world relevance of the task. One might suspect that these differences in goals stem merely from differences in modeling methodology. However, when Konrad writes a paper using the same methodology as Gunnar (i.e. linear systems), he cares most about the model being the optimal solution to a computational problem. Thus, diversity of modeling goals appears to be real.

\section{A short list of modeling goals}

To the extent that the goals we choose for modeling matter, an important open question is: what exactly are these goals? Examining a broad range of papers in computational neuroscience, we gleaned a variety of different modeling goals, typically revealed in the Introduction and Discussion sections. While it is impossible to produce an exhaustive list, we compile here a list of the most salient and common ones:

- Useful. Some models of the nervous system are also good at solving real-world problems. For example, a model of the visual system might be able to solve challenging problems in computer vision.

- Normative. Some models provide the optimal solutions to problems that exist in the real world. They are thus often used in domains where behavior or neural properties are expected to be optimal or near optimal. Thus, we might ask whether a model supplies the optimal solution to a computational problem faced by the brain.

- Clinically relevant. Some models produce insights that are relevant for developing or evaluating clinical interventions. Given the potential to reduce human suffering, there is no doubt that clinical relevance is a meaningful goal.

- Inspire experiments. Some models change the way we think about a problem and thereby raise interesting new hypotheses. This can then inspire new experiments.

- Microscopic realism. Some models describe the microscopic properties of the brain, such as synaptic, pharmacological, and cellular-level properties.

- Macroscopic realism. Some models describe properties of brain areas and networks. Addressing phenomena at this level may be attractive to many neuroscientists.

- Behavioral realism. Some models can faithfully describe and explain behavioral phenomena.

- Representational. Some models aim to use representations of information that are similar to representations in the brain. 
- Compact. Some models can be succinctly expressed in mathematical language and/or computer code. This makes these models particularly easy for humans to understand.

- Analytically tractable. Some models are understandable through mathematical equations as opposed to numerical simulations. For scientists with mathematical training, this provides a more generalizable understanding compared to numerical models.

- Interpretable. Some models can be easily interpreted with respect to how they work (e.g. what outcomes they predict) and/or how the brain might implement the computations (e.g. the underlying causal mechanisms).

- Beauty. Some models may be symmetrical, balanced, or resonate well with the way we think. This ephemeral quality is often a factor when assessing the merit of a model.

\section{Goals matter to how modeling is done}

The choice of goals matters in just about every imaginable way for modeling in neuroscience. Modeling goals affect the overall utility and interpretation of a model by influencing the evaluation metrics, the choice of model type, and the way we replace models with newer, better models. For example, if microscopic realism is required, this severely constrains the types of modeling techniques that can be used. Some research fields have implicit agreements on a set of desirable modeling criteria. For instance, historically, the eye-movements field has used linear-systems theory to model saccades: the field has been most concerned with behavioral realism, usefulness, inspiration of experiments, and interpretability, but has not placed much value on microscopic realism. If the field had been concerned with microscopic realism, the linear-systems approach would have likely been inappropriate and a different toolset-such as spiking neural-network models-would have been used instead. Thus, getting clarity on why we model may be just as important as understanding the mechanics of how to model.

\section{A simple survey demonstrates computational researchers have diverse goals}

To empirically assess modeling goals, we conducted an informal online survey in which we asked authors to rate their own modeling work with respect to the goals listed above. Participants rated up to 3 of their authored papers on each of the 12 goals, indicating the importance of each goal. We obtained results from 113 distinct authors who rated a total of 251 papers (Figure 1). On average, interpretability was rated as the most important modeling goal, whereas clinical relevance was rated as least important (Figure 1B, black bars). In addition, we found large variance of ratings across papers (Figure 1B, gray error bars), suggesting that there is, indeed, wide diversity of modeling goals in the neuroscience community. Not surprisingly, some goals are highly correlated (Figure $1 \mathrm{C}$ ), such as compactness and tractability. 


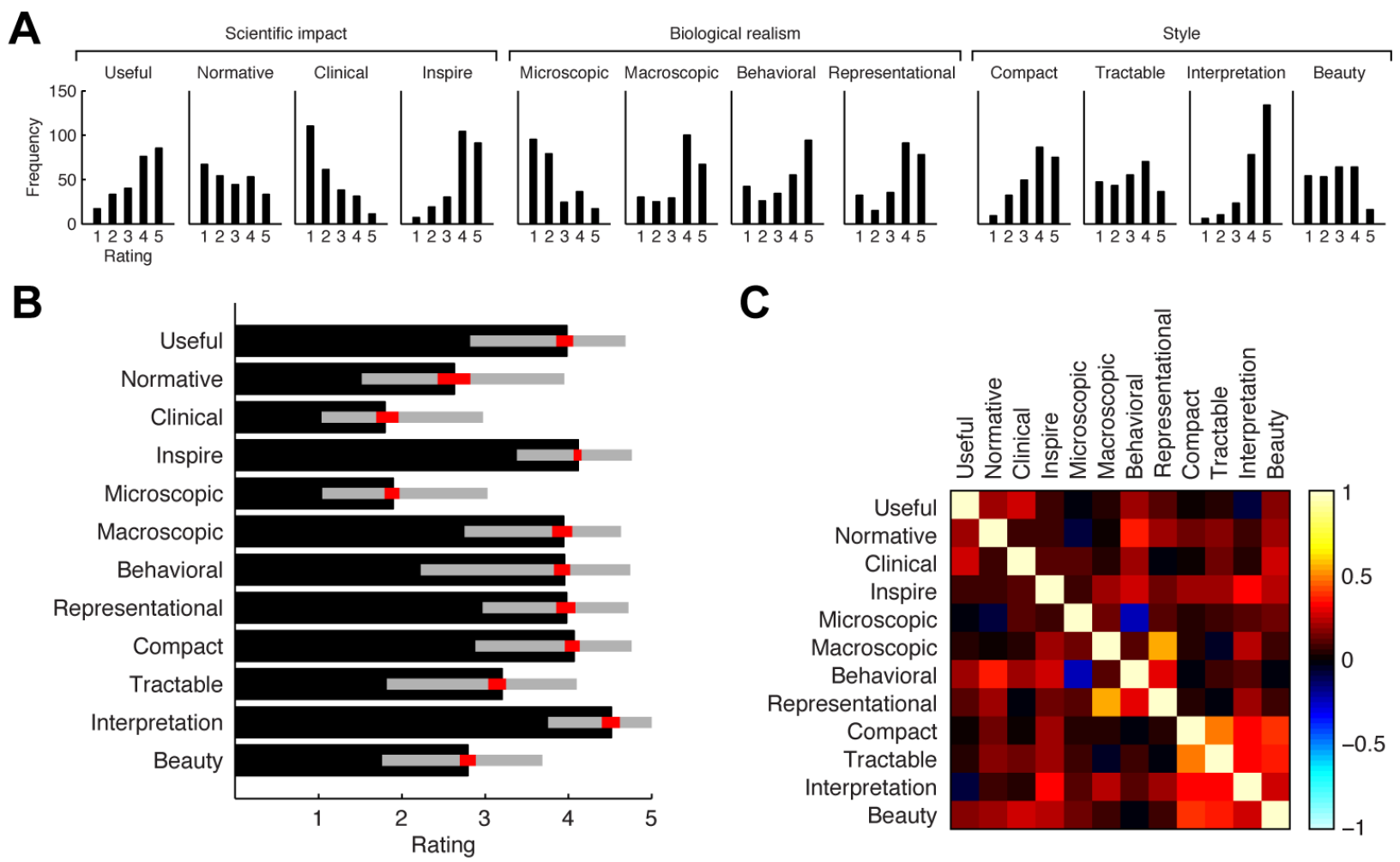

Figure 1. Modeling goals in the computational neuroscience community. We conducted an informal survey on the Internet to assess the modeling goals that different researchers held for specific papers that they authored (see Methods). (A) Histogram of results. For each of 12 modeling goals (dimensions), we plot a histogram of the reported ratings. Values range from 1 (completely irrelevant) to 5 (absolutely essential). Dimensions are ordered according to three conceptual groups that seem intuitively reasonable. (B) Summary statistics. For each dimension, we plot the median (black bars), interquartile range (gray error bars), and bootstrapped $68 \%$ confidence interval on the median (red error bars). (C) Pairwise correlation (Pearson's $r$ ) of dimensions across all papers.

To better understand the underlying structure of the ratings, we subtracted the average rating of each modeling goal and identified a lower-dimensional space using probabilistic principal components analysis (Figure 2A). We reconstructed the data in this lower-dimensional space and recomputed the pairwise correlation structure (Figure 2B). Finally, we re-ordered the modeling goals, revealing three groups (Figure $2 \mathrm{C}$ ). These groups overlap with the initial intuitive grouping of goals (as shown in Figure 1A), but not perfectly. One simple interpretation is that researchers vary along three different sets of modeling goals. This variation might reflect different subfields of neuroscience having distinct goals, or different types of modeling studies optimizing distinct criteria. However, this lower-dimensional reconstruction only accounted for $51 \%$ of the total variance in goal ratings. The remaining $49 \%$ of the variance reflects diversity of individual preferences in goals. Thus, just like the contrast between Gunnar's and Konrad's linear-systems models, the variability in modeling goals between researchers in general is high. 
A

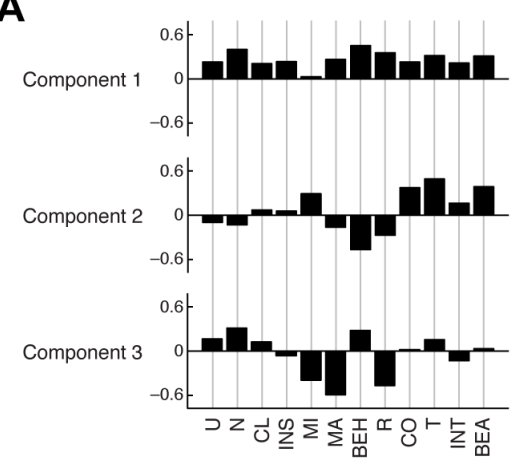

B

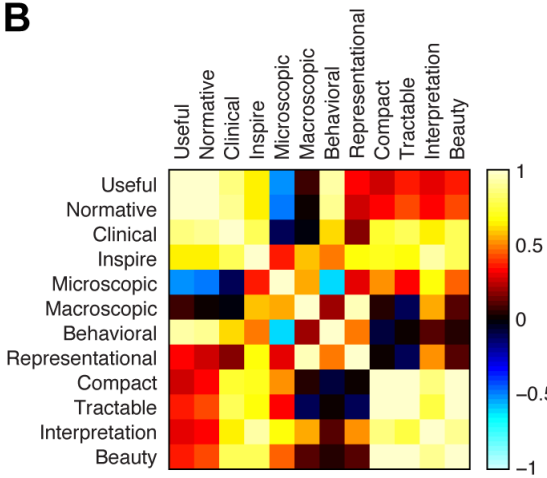

C

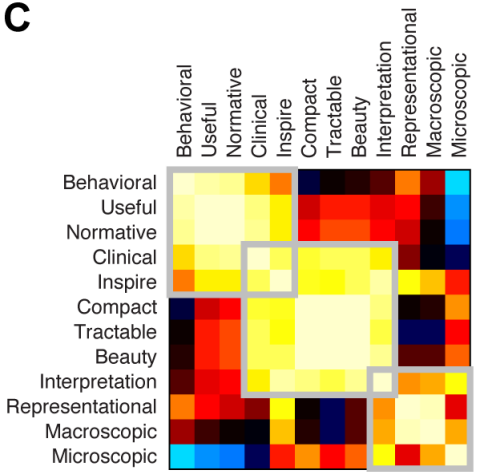

Figure 2. Groups of modeling goals. We performed probabilistic principal component analysis to characterize the latent covariance structure of the survey ratings. (A) Component loadings. The top three components (composing an orthonormal basis) are shown; the order of dimensions is the same as in Figure 1. (B) Data reconstruction. We reconstruct the original data using the three identified components and recompute the pairwise correlation as in Figure 1C. The correlation structure is similar to that of the original data, but stronger due to the dimensionality reduction. (C) Grouping of dimensions. We re-plot the results of panel B, re-ordering dimensions to highlight the block structure. Thick gray squares indicate three groups of dimensions that appear to be present in the data.

We acknowledge several limitations of the survey. The survey is neither a comprehensive nor random sample of computational neuroscientists. It is not calibrated nor validated, and the survey questions themselves may not have been interpreted in the same way by different participants. In the future, it may be fruitful to invest in more systematic meta-scientific analyses. For example, one might collect data from larger samples of participants, compare the modeling goals of different researchers who co-authored the same paper, compare whether a researcher can accurately predict the modeling goals of another researcher, and so on. Nonetheless, the current set of data is rich and can be used for interesting explorations (Figure 3).
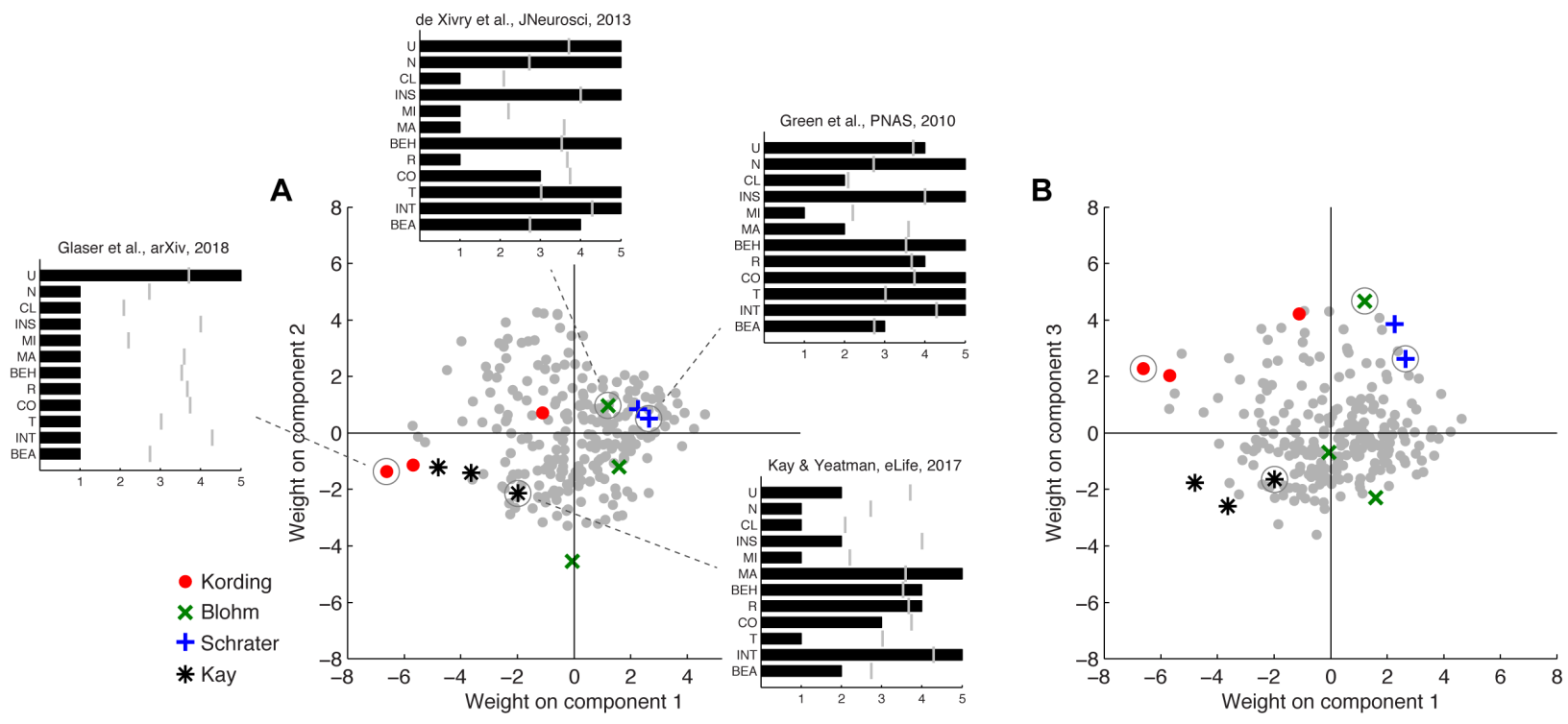
Figure 3. Diversity of modeling goals within and across authors. Here we plot all recorded paper ratings in the space defined by the first three principal components (see Figure 2A). (A) Space defined by components 1 and 2. Each gray dot indicates a single paper, and colored markers indicate papers from the authors. Insets show raw ratings for example papers; in these plots, thin gray lines indicate the mean of each dimension across all papers. Some authors are consistent across papers they write (Schrater, Kay), whereas other authors show more diversity in their papers (Kording, Blohm). Furthermore, there is high diversity in modeling goals across the four authors of this paper. (B) Space defined by components 1 and 3. Same format as panel A.

\section{Unification of goals?}

Modeling aims to generate insight into a phenomenon of interest. Since models in computational neuroscience all refer to brains, one could argue that they are guaranteed to produce synergistic answers and that the distinctions highlighted in this paper are not that important. We think this stance is debatable. Models positioned at different levels of biological realism (microscopic, macroscopic, behavioral, representational) are not guaranteed to inform each other, as distinct phenomena may emerge at different levels. Models following different styles (compact, tractable, interpretation, beauty) are optimizing different criteria, so a model that fulfills one criterion may be suboptimal under other criteria. Models aimed towards a specific type of scientific impact (useful, normative, clinical, inspire) often fail to deliver other types of impact. Thus, it is our contention that modeling goals are truly diverse and that models in computational neuroscience are not aimed towards a single coherent class of answers.

\section{Authors should explicitly state goals; readers should evaluate based on those commitments}

Why does it matter that different researchers have different modeling goals? Diversity often leads to significant tension and misunderstandings between researchers. For example, a reviewer might have a certain set of goals associated with a particular modeling approach and might evaluate a given paper outside of the authors' intentions. This out-of-scope evaluation is one of the most frequent and frustrating reasons for miscommunication in computational neuroscience. Its consequence is often paper rejection. We argue that this behavior is detrimental for science.

What are some practical steps we can take? As action items, we suggest that (1) authors should explicitly spell out their goals and (2) readers should deliberately evaluate a given paper within those constraints. For example, an author might include a section that begins, "In this paper, we sought to satisfy the criteria of $X, Y$, and $Z$. It is not our goal to develop a model that exhibits properties $A, B$, and $C$ for the following reasons..." We believe that explicit characterization of goals leads to more constructive interactions and therefore promotes scientific progress, discovery, and societal impact. 


\section{Methods}

To assess modeling goals in the computational neuroscience community, we constructed an online survey using Google Forms. Each of the authors then contacted colleagues via personal e-mails, mailing lists, and Twitter. We collected survey responses for approximately a month, with a survey deadline of August 31, 2018.

The survey asked each participant to choose up to 3 papers they authored or co-authored and to rate each paper on the 12 modeling goals described in the main text. The full set of survey questions and survey results are available at https://osf.io/pqe7f/. We note that the survey results may be useful for answering a variety of additional questions not addressed in this paper. For example, one might be interested to compare one's prediction of the modeling goals held by a given researcher to the actual goals held by that researcher. Or as another example, one might be interested to see where one's goals fall relative to the group norms.

For the analyses performed in this paper, ratings were aggregated across papers (251 papers from 113 distinct authors). For Figure 1B, a small amount of Gaussian noise (mean 0, standard deviation 0.5) was added to the data prior to computing summary statistics in order to avoid discretization effects. 